\title{
"Apneas" in Purkinje cell activity: evidence for the bistability of membrane potential in the awake cat cerebellum
}

\author{
Hugo Merchant* \\ Instituto de Neurobiología, Universidad Nacional Autónoma de México, Querétaro, México \\ *Correspondence: merchant@inb.unam.mx
}

\section{A commentary on}

Pausing Purkinje cells in the cerebellum of the awake cat

by Michael M. Yartsev, Ronit Givon-Mayo, Michael Maller and Opher Donchin

Sustained neural activity can be the result of either the dynamics of recurrent neural networks or the intrinsic properties of individual cells. In the later case, it has been shown in different brain areas that neurons can present a bistable behavior where brief inputs produce a prolonged increase in discharge rate due to a depolarized membrane potential. Neurons in this active condition, the "upstate", can switch to a hyperpolarized/downstate where they do not fire (O'Donnell and Grace, 1995). In vitro studies in different species have shown that Purkinje cells (PCs) of the cerebellum posses the intrinsic machinery to be considered bistable (Williams et al., 2002). The bistability of membrane potential is manifested in two firing profiles, namely, tonic firing of simple spikes (SSs) in the upstate and pausing periods in the down-state (Loewenstein et al., 2005; Schonewille et al., 2006). These neurons can toggle between both states spontaneously or after brief but significant stimulation in in vitro as well as in anesthetized in vivo preparations using intra and extracellular recordings. However, a recent discrepancy has emerged regarding whether or not PCs in awake animals can toggle from a preponderant up-state to the hyperpolarized condition (Schonewille et al., 2006). Yartsev et al. (2009) confronted these issues using extracellular recordings in the awake, behaving cat. They demonstrated that half of the PCs show long pauses in SSs activity that switched back and forth with periods of continuous firing. These cells, called pausing PCs (PPs), were clearly distinguished from continuous firing PCs using the following quantitative methods: (i) the coefficient of variation in the inter-spike interval (ISI); (ii) the dip test for unimodal instantaneous firing rate; and (iii) a bi-exponential fit to the ISI histogram - only in PPCs two distinct exponentials could be obtained (see Figures 1A (IV), B (IV)). The "apneas" in PCs activity seem to be independent of inhibitory inputs, and they show a duration that is similar to the periods of hyperpolarization observed in intracellular recordings. Hence, bistability inferred indirectly by extracellular recordings may be present in a large portion of PCs in the awake cat.

PCs exert an inhibitory influence in the deep cerebellar nuclei, and also receive excitatory inputs from both parallel and climbing fibers. In the absence of synaptic input, PCs can fire SSs spontaneously, however, with activation from climbing fibers they can generate complex spikes (CSs) (Ito, 1984). Part of the bistability controversy is whether the up/ down-state of PCs can be controlled by sensory evoked climbing fiber inputs in the behaving animal. Loewenstein et al. (2005) found in the anesthetized rat and guinea pig that PCs can switch between the up- and down-states after sensory activation of the climbing fiber input. Using similar intra and extracellular recordings, Schonewille et al. (2006) described that bistability can only occur infrequently in the awake mouse and that it can barely be influenced by natural sensory stimulation, motor performance or motor learning, whereas it is clearly present under anesthesia. Thus, a crucial question is whether or not anesthesia is a key player in the expression of bistability in PCs. The recent study of Yartsev et al. (2009) changed the balance toward the hypothesis of the functional link between the input from climbing fibers and the toggling in the bistability state of PCs. They demonstrated that in most of the PPCs, $25 \%$ of the state transitions from the continuous firing to the pausing state and vice versa were associated with the presence of CSs. Of course, these results are no evidence for a causal relationship between CSs and the PCs' state transitions in and out of pauses, although, their simultaneous occurrences were well above chance. More relevant is the fact that these authors did not find that cat behavior could toggle the SS firing rate. Therefore, the functional role of the bistability of PCs is still an open question.

Overall, the study of Yartsev et al. (2009) is providing strong evidence in favor of the idea that pauses in the activity of PCs can be relevant for information processing in the cerebellum. Needless to say, future research is required to determine the functional relevance of the pauses in PCs in mediating the cerebellar output during a number of behavioral contexts including motor performance and motor learning. These experiments will be technically challenging, since numerous electrode assemblies are needed in the cerebellar cortex and deep cerebellar nuclei to properly tackle this interesting problem in behaving animals.

\section{REFERENCES}

Ito, M. (ed.) (1984). The Cerebellum and Neural Control. New York, Raven Press.

Loewenstein, Y., Mahon, S., Chadderton, P., Kitamura, K., Sompolinsky, H., Yarom, Y., and Hausser, M. (2005). Bistability of cerebellar Purkinje cells modulated by sensory stimulation. Nat. Neurosci. 8, 202-211.

O’Donnell, P., and Grace, A. A. (1995). Synaptic interactions among excitatory afferents to nucleus accumbens neurons: hippocampal gating of prefrontal cortical input. J. Neurosci. 15, 3622-3639.

Schonewille, M., Khosrovani, S., Winkelman, B. H., Hoebeek, F. E., De Jeu, M. T., Larsen, I. M., Van der Burg, J., Schmolesky, M. T., Frens, M. A., and De Zeeuw, C. I. (2006). Purkinje cells in awake behaving animals operate at the upstate membrane potential. Nat. Neurosci. 9, 459-461.

Williams, S. R., Christensen, S. R., Stuart, G. J., and Hausser, M. (2002). Membrane potential bistability is controlled by the hyperpolarization-activated current $\mathrm{I}(\mathrm{H})$ in rat cerebellar Purkinje neurons in vitro. J. Physiol. 539, 469-483.

Yartsev, M.M., Givon-Mayo, R., Maller, M., and Donchin, O. (2009). Pausing Purkinje cells in the cerebellum of the awake cat. Front. Sys. Neurosci. 3, 1-9. doi: 10.3389/ neuro.06.002.2009.

Received: 15 October 2009; published: 15 April 2010. Citation: Front. Neurosci. (2010) 4, 1: 6. doi: 10.3389/ neuro.01.004.2010

Copyright $\odot 2010$ Merchant. This is an open-access publication subject to an exclusive license agreement between the authors and the Frontiers Research Foundation, which permits unrestricted use, distribution, and reproduction in any medium, provided the original authors and source are credited. 